\section{Effekt, statistischer}

R.-D. Hilgers ${ }^{1}$, N. Heussen ${ }^{1}$ und S. Stanzel ${ }^{2}$

${ }^{1}$ Institut für Medizinische Statistik, Universitätsklinikum der RWTH Aachen, Aachen, Deutschland

${ }^{2}$ Heidelberg, Deutschland

Synonym(e) Effekt; Statistischer

Englischer Begriff effect

Definition Als Effekte werden die Komponenten in statistischen Modellen ( $\triangleright$ Modell, statistisches), wie etwa dem Modell der $>$ Varianzanalyse, bezeichnet.

Beschreibung Im Rahmen einer Varianzanalyse erfassen die Effekte die Hauptwirkung und $>$ Wechselwirkung von Einflussfaktoren, deren Ausprägungen in Kategorien vorliegen. So kann z. B. für den beobachteten Wert der Zielvariablen (z. B. ein Laborparameter) bei 2 Einflussfaktoren A (z. B. verschiedene Labore) und B (z. B. verschiedene Messmethoden) das folgende statistische Modell gelten:

$$
\mathrm{Y}_{\mathrm{ijk}}=\mathrm{m}+\mathrm{a}_{\mathrm{i}}+\mathrm{b}_{\mathrm{j}}+(\mathrm{ab})_{\mathrm{ij}}+\mathrm{e}_{\mathrm{ijk}}
$$

wobei $m$ das Versuchsmittel und die $\mathrm{e}_{\mathrm{ijk}}$ die Fehlerkomponenten der einzelnen Beobachtungen ( $\triangleright$ Beobachtung) bezeichnen.

Von den übrigen im Modell auftretenden Größen, die Effekte genannt werden, dienen die $\mathrm{a}_{\mathrm{i}}$ zur Erfassung der Hauptwirkung des Faktors A, die $b_{j}$ zur Erfassung der Hauptwirkung des Faktors B und die (ab) ${ }_{\mathrm{ij}}$ zur Erfassung der Wechselwirkung beider Faktoren. Dabei heißt $\mathrm{a}_{\mathrm{i}}$ der $>$ Haupteffekt der i-ten Stufe (Ausprägung) von $A, b_{j}$ der Haupteffekt der $\mathrm{j}$-ten Stufe von B und $(\mathrm{ab})_{\mathrm{ij}}$ der $>$ Wechselwirkungseffekt zwischen der i-ten Stufe von A und der j-ten Stufe von B. Charakteristisch an dem obigen Modellansatz ist die Annahme, dass sich die einzelnen Effekte additiv zu einem Gesamteffekt ergänzen.

Mit der Methode der Varianzanalyse können diese Effekte geschätzt ( $\triangleright$ Schätzer) und Hypothesen über interessierende Effekte getestet $(\triangleright$ Test, statistischer) werden.

\section{Literatur}

Rasch D (1988) Biometrisches Wörterbuch. Verlag Harri Deutsch, Frankfurt am Main 